\title{
REPENSAR LA INTEGRACIÓN ENERGÉTICA SUDAMERICANA FRENTE A LA INCERTIDUMBRE DE LA INTEGRACIÓN REGIONAL Y LA TRANSICIÓN ENERGÉTICA GLOBAL
}

\section{RETHINKING SOUTH AMERICAN ENERGY INTEGRATION FACING UNCERTAINTY OF REGIONAL INTEGRATION AND GLOBAL ENERGY TRANSITION}

\author{
Ana Lía del Valle Guerrero
}

\begin{abstract}
RESUMEN
Este artículo tiene como objetivo analizar la integración energética en la Región Sudamericana entre 2004-2019, no de forma aislada, sino en su interrelación con la integración política/económica entre Estados y la transición energética como categoría transversal, a fin de incorporar la dimensión ambiental. Se considera que la energía es la base del desarrollo económico, pero, según sus fuentes, impacta en dos elementos contextuales claves de la problemática energética contemporánea: cambio climático y desarrollo sostenible. La metodología se fundamenta en una estrategia teórico-metodológica multimétodo 0 cualicuantitativa que, junto a la multiescalaridad y la multidimensionalidad como estrategias metodológicas, permiten analizar actores sociales más allá de una única escala de acción para ofrecer una visión más completa y compleja del objeto de estudio. Se emplean resultados obtenidos en investigaciones previas, complementados con revisión bibliográfica e interpretación de datos de informes de organismos internacionales. Se pone a discusión analizar los procesos de integración energética como productos de una realidad - cambiante e incierta - con complejas dinámicas en los procesos sociales, políticos y económicos, que influyen en el logro de un desarrollo energético regional sostenible.
\end{abstract}

Palabras Clave

Integración energética, Región Sudamericana, transición energética, cambio climático, desarrollo sostenible

\begin{abstract}
This article aims to analyze energy integration in the South American Region, between 2004 2019, not in isolation, but in its interrelationship with political/economic integration and energy transition as a transversal category, in order to incorporate the environmental dimension. Energy is considered the basis of economic development, but, according to its sources, it impacts two key contextual elements of contemporary energy issues: climate change and sustainable development. Methodology is based on a multi-method or qualitative-quantitative theoretical-methodological strategy. That together with multiscalarity and multidimensionality as methodological strategies, allow analyzing social actors beyond a single scale of action, to offer a more complete and complex vision of study object. Results obtained in previous investigations, are used and complemented by bibliographic review and interpretation of data from reports of international organizations. For discussion, analyze the energy integration processes as products of a reality - changing and uncertain - with complex dynamics in the social, political and economic processes that influence the achievement of sustainable regional energy development.
\end{abstract}

\section{KEYWORDS}

Energy integration, South American Region, energy transition, climate change, sustainable development 


\section{INTRODUCCIÓN}

En el mundo de comienzos del siglo XXI, el capitalismo global actual no tiene lógicas autónomas ni tampoco una sola lógica dominante que gobierne sobre las demás, sino que existen procesos complejos, heterogéneos y múltiples con diferentes temporalidades, dentro de un solo sistema-mundo de larga duración. Así, se observa cómo se tensionan los tiempos históricos en contextos geopolíticos dinámicos y complejos, que rompen la linealidad de los diferentes procesos de integración, presentando avances y retrocesos, principalmente, en sus aspectos políticos y económicos que impactan en la sociedad y en el logro de la integración (Guerrero, 2019b).

En este sentido, en relación con la integración energética en particular, como sostiene Carrizo (2019)

Entre finales del siglo $X X$ y principios del siglo $X X I$ se produce un vaivén en los procesos de integración energética regional, con momentos de extremo dinamismo basados en la explotación de los hidrocarburos, en particular el gas, con interconexión de redes nacionales, seguidos luego por grandes proyectos de energía en América del Sur y, otros momentos, donde se producen reveses en los intercambios y, en la evolución de los mismos (p.175).

A fin de contextualizar la situación de la integración energética en su imbricación con los procesos de integración política y económica en la Región Sudamericana, se analizan cambios producidos en los últimos años entre 2004 y 2019, desde una mirada multiescalar y multidimensional, que considera puntos de vista económicos, políticos, ideológicos, sociales y ambientales que impactaron en los procesos de integración regional en dos periodos consecutivos. Un primer período entre 2004-2014, caracterizado por la presencia de gobiernos progresistas que refuerzan procesos de integración regional, más desde lo político/ideológico que desde lo económico/social, ya que se apoyan en la afinidad ideológica entre sus líderes (Argentina, Brasil y Venezuela).

En el segundo periodo, desde el año 2015 y aún en construcción, se observa un cambio de liderazgo regional: la Argentina y Brasil pierden la iniciativa, mientras que Chile y Colombia encabezan cambios de orden geopolítico y económico propiciando la creación del Foro para el Progreso de América del Sur (PROSUR) en reemplazo de la 
Unión de Naciones Sudamericanas (UNASUR) y se produce el avance de la Alianza del Pacífico frente al Mercado Común del Sur (MERCOSUR). Estos cambios en los organismos políticos y económicos de integración regional, en 2020, aparecen más como cuestiones coyunturales que como cambios estructurales.

A este contexto de incertidumbre en las dimensiones políticas, ideológicas y económicas, se suma, a fines de 2019 , la dimensión social con la denominada "primavera sudamericana" con un aumento de las demandas de las nuevas clases medias que cuestionan las instituciones por una baja confianza en las mismas, reclamando mejoras a los Estados de sus servicios de salud, educación y seguridad social. Como consecuencia de estas movilizaciones sociales, la transformación del mapa político regional pasa desde un enfoque ideológico de enfrentamientos izquierda/derecha a una nueva divisoria de corte sociopolítico, basado en reclamos sociales con un "voto castigo" que define las elecciones en la región.

En ese marco de reclamos sociales, en marzo de 2020 emerge un evento disruptivo a escala global, la pandemia del COVID-19, que desnuda aún más las debilidades del sistema de salud y las desigualdades sociales a escala regional. En la búsqueda de soluciones que todavía continúan al momento de escribir el artículo, se observa por un lado acciones de los Estados que buscan enfrentar un problema común de modo conjunto y, por otro, simultáneamente, un cierre de fronteras y un aislamiento de cada país sobre sus propias agendas internas bloqueando en parte avances de los mecanismos de integración.

Desde esta trama ampliada, el artículo estudia la cuestión crucial de la integración energética desde una mirada multidimensional y multiescalar de la energía. Tiene como objetivo analizarla en la Región Sudamericana entre 2004 - 2019, no de forma aislada sino en su interrelación con la integración política/económica entre Estados y la transición energética como categoría transversal, a fin de incorporar la dimensión ambiental. En este sentido, se considera que la energía es la base del desarrollo económico pero, según sus fuentes, impacta en dos elementos contextuales claves de la problemática energética contemporánea: cambio climático y desarrollo sostenible.

La metodología se fundamenta en una estrategia teórico-metodológica multimétodo o cualicuantitativa que, junto a la multiescalaridad y la multidimensionalidad como estrategias, permiten analizar actores sociales más allá de una única escala de acción, 
a fin de ofrecer una visión más completa y compleja del objeto de estudio. Se emplean resultados obtenidos en investigaciones previas de la autora y se complementa con revisión bibliográfica e interpretación de datos obtenidos de informes elaborados por organismos internacionales en relación directa con la temática analizada.

El marco teórico adoptado parte del enfoque de la Nueva Geografía Política, que apunta al estudio del Estado a través de las relaciones de poder en el espacio a distintas escalas. Se analiza al espacio como producto de un sistema multiescalar y multidimensional, en el cual se dan relaciones políticas junto a relaciones de poder (Guerrero,2016). Esta perspectiva multiescalar permite un acercamiento a la realidad sudamericana en su interacción con las escalas global, regional, nacional y local, a la vez que visibiliza tendencias a la integración o a la fragmentación territorial de acuerdo a las decisiones políticas adoptadas por los Estados. A su vez, la perspectiva multidimensional permite un abordaje más integral al considerar las dimensiones políticas, económicas, sociales y ambientales en forma simultánea.

Abordar la cuestión energética desde este enfoque se encuentra vinculado más que a un abordaje determinado desde la perspectiva del tamaño, de la jerarquía/ nivel, a una concepción relacional que implica que las escalas no pueden ser concebidas como instancias aisladas sino como resultado de un complejo de relaciones sociales y económicas transescalares que las interpenetran, configuran y transforman permanentemente (Fernández, 2010; Howitt, 1998).

El análisis de la integración energética desde una mirada multidimensional de la energía considera, como sostienen Recalde y Guzowsky (2016), que la energía tiene diferentes dimensiones: a) dimensión económica, por la relación que existe entre consumo, actividad económica y su incidencia en el precio de los productos; b) dimensión social, ya que el consumo de energía es relevante para el desarrollo social, el acceso a la energía es considerado una de las principales políticas para la reducción de la pobreza; c) dimensión política, pues la energía es un bien estratégico para el desarrollo socioeconómico; d) dimensión geopolítica, por la importancia para el logro de la seguridad energética de un país; e) dimensión ambiental, que se vincula con el ambiente a diferentes escalas y, en particular, con el cambio climático.

En relación con este último punto, el Panel Intergubernamental de Cambio Climático 2014 (IPCC, por sus siglas en inglés) afirma que las emisiones de gases del sector 
energético representan más del $30 \%$ de las emisiones totales de gases de efecto invernadero. De allí surge la propuesta de estudiar la integración energética sudamericana en el marco de la transición energética global, considerando que se debe lograr incluyendo a la transición energética como factor fundamental para alcanzar un desarrollo sostenible, con objetivos más amplios que contengan la dimensión social, económica y ambiental a fin de garantizar la equidad social y el bienestar para la sociedad en su conjunto.

En este marco general, el artículo se estructura en cuatro apartados: a) Breve reseña de la integración política y económica sudamericana; b) Transición energética, cambio climático y desarrollo sostenible; c) Integración gasífera e Integración eléctrica en Sudamérica; d) Incertidumbres de la transición energética sudamericana en el contexto de la integración energética regional. El esquema de la Figura 1 constituye una propuesta interpretativa de los vínculos existentes entre los diferentes procesos de integración seleccionados para el análisis y la transición energética como categoría transversal, en el contexto del cambio climático y del desarrollo sostenible.

Figura 1. Propuesta interpretativa de los procesos seleccionados de la Integración regional sudamericana

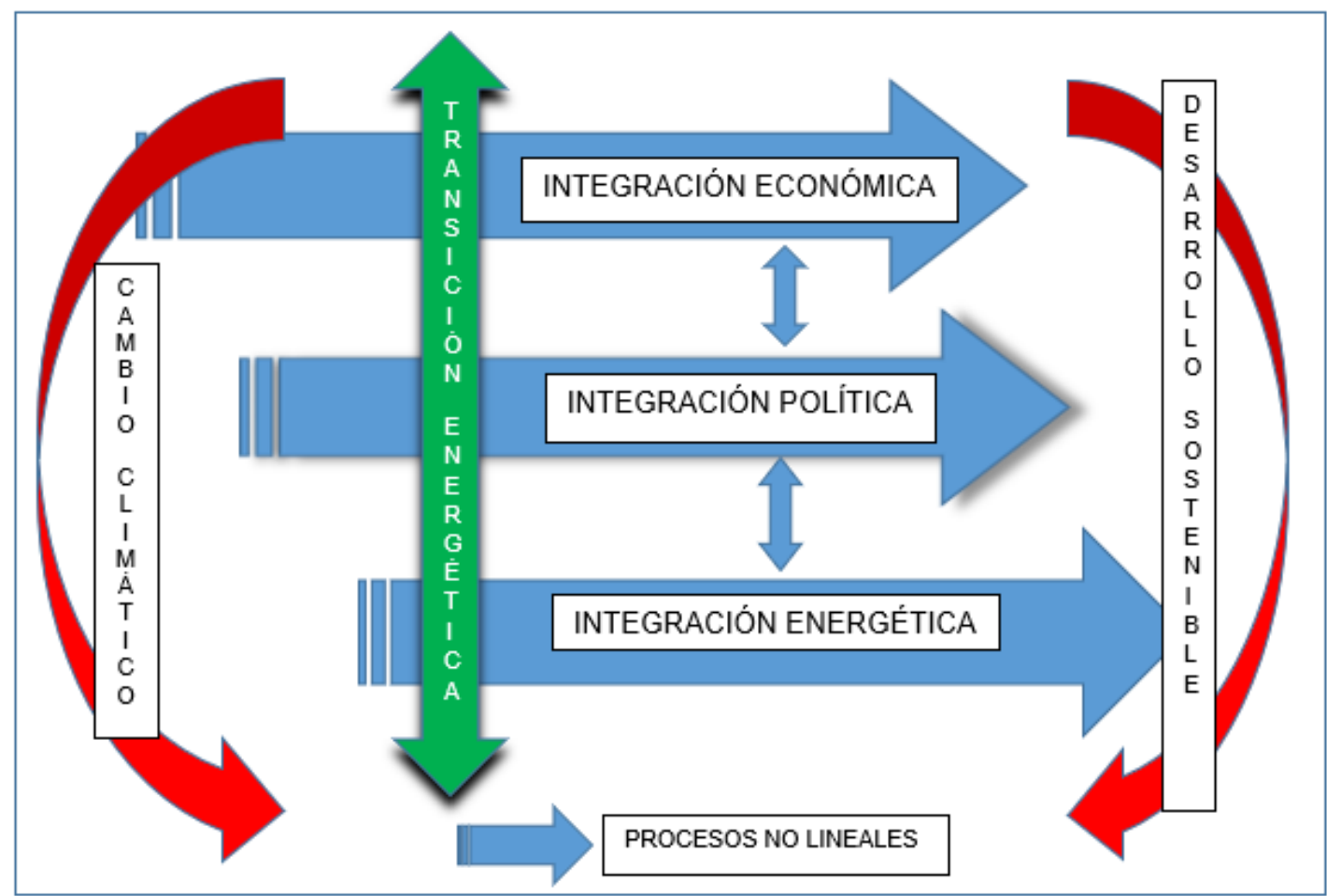

Fuente: Guerrero, 2020 


\section{BREVE RESEÑA DE LA INTEGRACIÓN POLÍTICA Y ECONÓMICA SUDAMERICANA}

Dentro del contexto descrito, desde una perspectiva Sudamericana, en la región se está produciendo una reconfiguración de los procesos de integración producto de entornos geopolíticos dinámicos y complejos que, a diferencia de otros momentos históricos, están regidos por cuestiones sociopolíticas más que por cuestiones económicas o militares. Ello se visibiliza en la Región Sudamericana, tanto en las diferentes fracturas -internas y externas- como en los avances y retrocesos que van desde la integración hemisférica a la integración latinoamericana y sudamericana, buscando articularse en cada momento histórico según los cambios de contexto/ciclos nacionales e internacionales (Guerrero, 2019b).

En esta encrucijada entre gobiernos progresistas y liberales, la UNASUR, como sostiene Sanahuja (2019), quedó paralizada como instancia de diálogo político y gestión de crisis a causa del enfrentamiento ideológico y los vetos cruzados del bloque "bolivariano" y los gobiernos de derecha. Además, menciona que en agosto de 2017 se creó el "Grupo de Lima" como mecanismo ad hoc para afrontar la crisis política y la deriva autoritaria en Venezuela, por lo que varios países decidieron abandonar UNASUR y establecer el PROSUR, en marzo de 2019, como nuevo marco de diálogo político entre gobiernos afines de derecha. Al mismo tiempo, la Alianza del Pacífico con una visión liberal-conservadora, también se enfrenta a un escenario más adverso y a sus propias contradicciones.

Sanahuja (2019) afirma también que un examen de conjunto de la integración regional y del regionalismo latinoamericano, y de sus vicisitudes más recientes, deja un balance poco halagüeño. Asimismo, advierte que este cambio no está aislado de los que se producen con un ciclo global de ascenso del nacionalismo y la extrema derecha que se observa tanto en Europa como en Estados Unidos y en otras latitudes, que no solo cuestionan la integración europea sino que también, en un sentido más amplio, impugnan el orden internacional liberal. Las nuevas derechas latinoamericanas apuestan por la globalización y la vinculación con las potencias centrales, aunque esta apuesta resulta tardía en el marco de los cambios nacionalistas en los países centrales (Sanahuja y Comini, 2018).

En el mismo sentido Tokatlian (2019) sostiene que, desde comienzos del siglo XXI, distintos gobiernos en Sudamérica reivindicaron el mérito de la integración, como lo 
demuestra el permanente relanzamiento del MERCOSUR, la reivindicación inicial de la UNASUR, la fundación del Alba, el establecimiento de la CELAC y la conformación de la Alianza del Pacífico. Sin embargo, el estado real de la integración en América del Sur es muy pobre y, en consecuencia, se puede afirmar que los cambios son cada vez más acelerados e inciertos.

De este modo, la Región Sudamericana pierde relevancia en la política mundial, con el debilitamiento de sus organismos políticos y económicos y, por lo tanto, dejan de contribuir al multilateralismo global y refuerzan el bilateralismo en sus acuerdos internacionales. En este sentido, se destaca como tanto los bloques económicos regionales (MERCOSUR; CAN; Alianza del Pacífico) como los políticos (ALBA, CELAC, UNASUR) pierden fuerza y se desarticulan frente al avance de acuerdos bilaterales ya sea con Estados Unidos, China o Rusia, mostrando estas fracturas externas e internas que en la actualidad debilitan las relaciones que potenciaban estos bloques regionales en la década pasada.

Se puede afirmar que, más allá del discurso integracionista de los diferentes gobiernos, el estado real de la integración en América del Sur es débil, en tanto en cada coyuntura política y económica se generan nuevos proyectos que compiten con otros previos que no llegaron a consolidarse. Al sumar al análisis de los procesos políticos y económicos de integración regional la dimensión social con las consecuencias de la primavera sudamericana y la crisis del COVID-19, al incorporar la dimensión ambiental con la transición energética, se observa la complejidad, heterogeneidad y diversidad de los procesos de integración entre los cuales se encuentra inserta la integración energética.

Con relación a ella, desde el punto de vista económico surge la pregunta de si el regreso de los gobiernos de derecha en distintos países de la región favorecerá procesos de mercantilización de los recursos, ya iniciados en la etapa anterior con gobiernos progresistas, como garantes de un nuevo proceso de concentración de los capitales rentísticos y financieros donde importa más la rápida monetización de las reservas que crear lazos que favorezcan la integración.

En este sentido, la integración física es la base para la económica a través de la infraestructura (energía y transporte). En la Región Sudamericana se refleja en la Iniciativa para la Integración Regional Sudamericana IIRSA/COSIPLAN, con foco en el 
desarrollo de infraestructura. A partir del año 2011 la Iniciativa IIRSA se incorpora al trabajo del Consejo Suramericano de Infraestructura y Planeamiento (COSIPLAN) de la UNASUR como su foro técnico para temas relacionados con la planificación de la integración física regional suramericana.

En relación con la integración energética en particular, el Plan de Acción Estratégico (PAE) para el período 2012-2022, se organiza alrededor del concepto de Ejes de Integración y Desarrollo (EID) mediante una Agenda de Proyectos Prioritarios de Integración (API); en esa agenda solo dos proyectos se dirigen al sector energía mientras que los 29 restantes corresponden al sector transporte. Estos números hacen evidente el estancamiento en los procesos de integración energética.

La interrelación entre la integración política, económica y energética, se visibiliza a través de las acciones tomadas en los diferentes ciclos de gobierno, quienes además de planificar y promover la red de infraestructura y energía en Sudamérica, apoyan estrategias de financiamiento, inversión, cambios y/o adecuación de los marcos legales, muchas veces a partir de acciones impuestas por actores globales con poder de decisión en el espacio regional y nacional.

Estas acciones favorecen la consolidación de ¿corredores de "integración" o de interconexión? como reflejo de las relaciones transescalares de poder, que muchas veces facilitan la extracción de recursos, a partir de la construcción de infraestructura física que favorece la extracción y exportación de los mismos sin que prevalezcan las necesidades regionales. Cabe preguntarse si a futuro, debido a los cambios políticos, la pérdida de peso de la UNASUR, donde se encuentra el COSIPLAN, también afectará a los proyectos de integración/interconexión energética, o si los Estados de la región tomaran decisiones propias que prioricen inversiones en procesos de desarrollo de energías renovables tradicionales y nuevas que favorezcan la transición energética y el desarrollo sostenible priorizando las necesidades regionales.

Por último, desde un punto de vista sociopolítico y en el contexto de la actual crisis causada por la pandemia del coronavirus, tal vez los gobiernos y la sociedad recuerden su dependencia de la electricidad y comiencen a revalorizar la importancia de disponer de este recurso en forma confiable y asequible. Como explican Hallack y Yepez (2020), la sociedad moderna depende de ella ya sea de forma directa en los hospitales (para operar ventiladores, refrigeradores y otros equipos médicos), en la 
comunicación entre sociedad y gobierno, o indirectamente para permitir una cierta continuidad de la producción (a través de esquemas de teletrabajo), educación (estudios on-line) y entretenimiento para sobrellevar el aislamiento o cuarentena (las personas conectadas a través de sus teléfonos, computadoras y otros aparatos electrónicos).

En este sentido, se revaloriza la importancia para el Estado de garantizar a la población el acceso a los servicios de energía. Pero ello depende de la operación y mantenimiento de la infraestructura gasífera y eléctrica, de la disponibilidad de combustibles, y también de la asequibilidad de los servicios para todos los usuarios, en particular para aquellos de menores ingresos.

\section{TRANSICIÓN ENERGÉTICA, CAMBIO CLIMÁTICO Y DESARROLLO SOSTENIBLE}

A los conflictos mencionados en las dimensiones política, económica y social, se agrega la dimensión ambiental en relación con el uso de las diferentes fuentes de energía que han ido cambiando a través del tiempo. Las transiciones energéticas marcaron estos cambios de acuerdo con los avances de la tecnología y la posibilidad para incorporarlos de cada sociedad, según su grado de desarrollo económico.

En relación con la transición energética, ésta se desarrolla durante largos períodos de tiempo - 40 a 130 años- y se relaciona con la transición de una economía con una fuente dominante de energía y su correspondiente tecnología a otra (Fouquet, 2012, p. 3). Se identifican hasta la actualidad tres principales transiciones energéticas.

La primera fue el paso desde la leña hacía el uso del carbón como fuente de energía dominante y coincide con la denominada Primera Revolución Industrial, con centro en Gran Bretaña, donde el desarrollo de la máquina de vapor dependía de la combustión sobre la base del abundante carbón que existía en el territorio inglés. A fines del siglo $\mathrm{XIX}$ y principios del siglo $\mathrm{XX}$, se produjo la segunda con el descubrimiento del motor de combustión interna y las necesidades de desarrollo de la industria, buscando la energía proveniente de combustibles derivados del petróleo como base de la actividad económica en coincidencia con la Segunda Revolución Industrial (Guerrero, 2016, sobre la base de OLADE, 2013).

La tercera es la contemporánea. Parte de un modelo basado en un alto consumo de energías fósiles con elevadas emisiones de gases de efecto invernadero que, de no 
revertirse, situarán al planeta ante escenarios de cambio climático y de incremento de la temperatura mundial respecto a la época preindustrial por encima de los $2 \stackrel{\circ}{\mathrm{C}}$, con consecuencias aún más graves. El cambio climático es un reto global que no respeta las fronteras nacionales y la energía es uno de los principales factores que contribuyen al mismo, ya que representa alrededor del $60 \%$ de todas las emisiones mundiales de gases de efecto invernadero. En este sentido, ya no es una amenaza en un horizonte lejano, sino que presenta un avance cada vez más rápido que requiere de una transición energética que evolucione hacia una economía descarbonizada y sostenible.

En este contexto, para fortalecer la respuesta global a la amenaza del cambio climático, los países adoptaron el Acuerdo de París en la COP21 en coincidencia con la Agenda de las Naciones Unidas para el desarrollo sostenible, con escenarios de medio-largo plazo y objetivos a 2030 y 2050, para evitar ese incremento de la temperatura mundial. Los países de América Latina y el Caribe también se han comprometido a establecer la Agenda 2030 como una política de Estado, articulando marcos institucionales para incorporar los 17 Objetivos del Desarrollo Sostenible (ODS) en sus planes y políticas nacionales de desarrollo, procurando alinear sus presupuestos nacionales, locales y sectoriales, para lo cual es clave que se definan políticas regionales y nacionales a favor de fuentes renovables que atiendan la futura demanda de energía (CEPAL, 2018).

La actual transición energética tiene la particularidad - frente a las que se produjeron hasta el momento- de que la tendencia no es hacia una fuente de energía dominante, sino que se emplean diferentes fuentes lideradas por el desarrollo simultáneo del gas y de las nuevas energías renovables (eólica y solar), denominadas así para diferenciarla de la energía hidroeléctrica por considerar que, a pesar de usar un recurso renovable como el agua, causa daño al ambiente al construir las represas para generar energía eléctrica.

Además, se concentran los mayores esfuerzos en el uso eficiente de los combustibles fósiles, en particular el gas, por ser un combustible de transición o puente, más abundante que el petróleo, de menor precio y menos contaminante que el carbón. Por ello, el informe BP 2019 denomina a la transición energética contemporánea como "transición energética dual", mientras que otro informe generado por la International Renewable Energy Agency (IRENA, 2019), a través de la Global Commission on the 
Geopolitics of Energy Transformation, con apoyo de Alemania, Noruega y Emiratos Árabes Unidos, la denomina como "transformación energética" puesto que implica un escenario más complejo e integrado que la sola transición energética.

Este último informe remarca que no es solo un cambio de un combustible a otro sino que son diferentes combustibles en uso, incorporándose a diferentes sectores, con distintas velocidades en cada país o región, motivo por el cual es una transformación mucho más profunda y compleja del sistema energético global que tendrá implicaciones sociales, económicas y políticas que van más allá del sector energético en particular (Guerrero, 2019 a). De esta breve contextualización de la transición energética global, surge que el principal fundamento de la contemporánea es la búsqueda de un sistema energético menos contaminante.

En este sentido, la firma del Acuerdo de París en la COP21 el año 2015 y la Agenda 2030 de la ONU, ofrecen una oportunidad sin precedentes para orientar al mundo en la senda del desarrollo sostenible y el cambio climático con sus objetivos finales de erradicar la pobreza, mejorar las condiciones de vida de la población y lograr la rápida transición a una economía baja en emisiones de carbono y resiliente al cambio climático. Ambos procesos se refuerzan mutuamente, si se implementan de manera conjunta y pueden promover la prosperidad y la seguridad de las generaciones presentes y futuras. La transición energética es el paso previo necesario.

La Agenda 2030 tiene por objeto abordar los problemas actuales y futuros a través de los ODS. En relación con la integración energética se deberían tomar como objetivos centrales los identificados 7,12 y 17. El Objetivo 7 (energía asequible y no contaminante) busca asegurar el acceso universal a los servicios de energía modernos, mejorar el rendimiento energético y aumentar el uso de fuentes renovables para crear comunidades más sostenibles e inclusivas y para la resiliencia ante problemas ambientales como el cambio climático.

En relación con ello, el Objetivo 12 (acción por el clima) es de carácter transversal, en el cual se pide que se eliminen los subsidios a los combustibles fósiles y otras distorsiones que fomentan el uso ineficiente de recursos y previenen la penetración de tecnologías e insumos más limpios ofreciendo, además, espacio fiscal para aplicar las políticas sociales necesarias (CEPAL, 2018, p. 56). 
Por último, en relación con el logro de la integración energética, el Objetivo 17 (Alianzas para lograr los objetivos) propone un programa exitoso de desarrollo sostenible que requiere alianzas entre los gobiernos, el sector privado y la sociedad civil. Estas alianzas inclusivas son necesarias a nivel global, regional, nacional y local, implican un mayor diálogo entre los diferentes actores, y deben ser construidas sobre principios y valores comunes, junto a una visión y metas compartidas que colocan a la gente y al planeta en el centro de interés. Sería deseable entonces que se alcanzara de este modo una transición energética justa y una integración energética sostenible.

\section{INTEGRACIÓN GASÍFERA E INTEGRACIÓN ELÉCTRICA EN SUDAMÉRICA}

Desde sus orígenes históricos, la creación del MERCOSUR en 1991 favoreció la integración regional y tuvo su capítulo energético en dos documentos (Memorando 10/98 y Memorando 10/99) donde se fijaron las pautas para la integración eléctrica y gasífera, tendientes a favorecer el libre comercio. Como sostiene Honty (2006), se buscaba una interconexión física para transportar electricidad y gas natural pero sin considerar cuestiones como eficiencia energética, cuidado del ambiente o desarrollo sostenible.

Esta contextualización previa de la situación de la integración política y económica regional junto a la transición energética, como categoría transversal que atraviesa los diferentes procesos de integración, muestra cómo se imbrican los diferentes procesos que influyen en el desarrollo de la integración energética. Dentro de la misma, los subsectores eléctrico y gasífero sudamericanos tienen una profunda relación entre sí puesto que las reservas de gas natural, gracias a los avances tecnológicos de las turbinas de ciclo combinado, se emplean como fuente para la generación eléctrica. Así, en el marco de la propuesta de repensar la integración energética sudamericana, los siguientes puntos profundizan el análisis de los procesos que se producen en estos subsectores.

Este análisis de ambos ejes se realiza desde un enfoque multidimensional y multiescalar de la integración energética regional, que se contextualiza temporalmente en el período 2004-2019, a fin de mostrar cómo los diferentes ciclos de gobiernos progresistas y liberales, con cambios tanto políticos como productivos, no incidieron en el desarrollo de la infraestructura eléctrica regional que aparece como un componente estable en el marco de los vaivenes de los procesos de integración energética 
regional. Mientras que, en el caso de la integración gasífera, se observa cómo las decisiones políticas y económicas de abastecimiento de gas en los países de la región, estuvieron condicionadas por conflictos geopolíticos, actuales e históricos aun irresueltos que llevaron a una mayor dependencia de actores extraregionales y a un estancamiento de la integración gasífera.

\section{III.1. INTEGRACIÓN GASÍFERA Y CONFLICTOS POLÍTICOS/GEOPOLÍTICOS}

En relación con el análisis de los conflictos que se generan en la región para alcanzar la integración gasífera, constituyen insumos para este trabajo los resultados obtenidos en investigaciones previas de la autora, junto a datos de CEPAL 2019. Para lograr el abastecimiento fluido de gas en la región, los Estados despliegan diferentes acciones que generan conflictos que avanzan desde un conflicto bilateral a una cuestión regional y luego global, actuandocomo obstáculos a la integración gasífera regional al incorporar actores extraregionales como China, Irán y Rusia, históricamente ajenos a la región.

En el período analizado, 2004-2019, se pueden delinear dos escenarios que muestran continuidades y rupturas en las relaciones de poder entre los Estados que impactan en la integración gasífera regional.El primero se extiende entre $2004-2007$, y el segundo entre 2008 - 2019 y aun continúa en desarrollo.

El conflicto por el abastecimiento de gas en la región surge en 2004 por la falta de cumplimiento de contratos firmados entre Estados, cuando la Argentina recorta las exportaciones de gas a Chile para asegurar el abastecimiento de su mercado interno. Esta situación señala que en sus inicios se trataba de un conflicto bilateral que luego se expande a la región. Hasta ese momento, desde lo político, la firma de acuerdos bilaterales de largo plazohabía sido la solución encontrada en la región para superar problemas de abastecimiento.

Los tratados firmados entre los Estados - visibilizados través de la construcción de gasoductos - forman parte de la infraestructura física que favorece la integración energética regional. Ejemplos de ellos son los firmados entre Bolivia y Brasil (contrato 1999-2019, prorrogado hasta 2023/2025 de acuerdo a volúmenes de consumo); Bolivia y la Argentina (primer contrato entre 1972 y 1999, luego suspendido y retomado entre 2004 - 2006 de forma temporaria y prorrogado por 20 años hasta 2026); o entre la Argentina y Chile (Protocolo de Integración Energética 1995). Este se 
incumple desde 2004 y en 2019 se retoma la exportación con el desarrollo de Vaca Muerta. Sin embargo, la firma de estos tratados no garantiza su cumplimiento efectivo ya que se observan fallas en su implementación que llevaron al surgimiento de conflictos.

En este contexto, se identifica un escenario inicial a escala regional, entre 2004-2007 en el cual se refuerzan las relaciones bilaterales y los procesos de integración gasífera que adquieren un carácter regional al incorporar otros Estados, como Bolivia y Perú, en la búsqueda de solución al conflicto bilateral a través de diferentes propuestas de construcción de gasoductos, que implican la firma de contratos de largo plazo.

Una de las propuestas de solución al conflicto fue a través de la creación de un anillo energético sudamericano, con centro en Perú - dejando de lado a Bolivia-, a partir de la explotación de los yacimientos de gas descubiertos en Camisea (Perú) que inician su producción en 2004.Proponía redireccionar los flujos hacia Chile y desde allí hacia la Argentina. Este proyecto no se concretó por tensiones geopolíticas existentes, que aún continúan, en cuanto a la delimitación de la zona económica exclusiva en el mar territorial entre Chile y Perú.

Este período coincide, en el año 2006, con un predominio de gobiernos progresistas en la Región Sudamericana, alineados en sus posturas ideológicas, políticas y económicas donde los núcleos de poder regional estaban encabezados por Venezuela y Brasil, con la formación de dos ejes respectivamente: la Alianza Bolivariana de las Américas (ALBA), más radicalizado en su posición y el Mercado Común del Sur (MERCOSUR), más flexible, que actúa como contención del eje anterior y como garante de la paz regional. Todo ello haría suponer mayores facilidades para avanzar en la integración energética regional.

En este marco, un segundo intento de solución se basó en un acuerdo entre Bolivia y Chile que intercambiaría gas por salida al mar. Se basaba también en los cambios políticos producidos con presidentes más cercanos en sus posturas políticas/ideológicas, Evo Morales en Bolivia y Michelle Bachelet en Chile, que permitiría dejar de lado sentimientos nacionalistas dando prioridad a la mejora de la calidad de vida de la población a través de un aprovechamiento de recursos que garantizarán el desarrollo económico nacional. Sin embargo, no llega a concretarse y Bolivia exporta gas hacia la Argentina con una claúsula explícita que decía que "ni una 
molécula de gas podía exportarse hacia Chile" como consecuencia de conflictos geopolíticos históricos, aun irresueltos, por la pérdida de salida al mar de Bolivia en la Guerra del Pacífico, con Chile y Perú (Guerrero, 2016).

Un tercer intento de solución surge desde Venezuela, con la propuesta del Presidente Hugo Chávez, de la construcción del denominado Gran Gasoducto del Sur, el cual daría fin a los problemas energéticos de la Argentina y Brasil e, indirectamente, solucionaría también los problemas de Chile y Uruguay. Este gran megaproyecto de 8000 Kilómetros de extensión -que no incluía a Bolivia- requería enormes inversiones en infraestructura. Estas oscilaban entre 16.000 y 20.000 millones de dólares y conectarían Puerto Ordaz en Venezuela, con Manaos en Brasil y luego, mediante gasoductos secundarios, se conectaría con Río de Janeiro y otros puntos en Brasil. Hacia el sur llegaría a Montevideo, incorporando a Uruguay y luego a Buenos Aires, en Argentina. Sin embargo, es un proyecto casi utópico por el costo, el tiempo, la cantidad de ambientes y países que debía atravesar y, además, todos con diferentes legislaciones.

Este proyecto surge de una competencia - cada vez mayor - por los espacios de influencia política regionalentre los ejes mencionados, donde nuevamente se entrecruzan cuestiones políticas y energéticas y la energía aparece como factor de disputa por el poder y no como factor de integración. En síntesis, en el período 20042007, los únicos gasoductos existentes y en funcionamiento conectan con Bolivia, mientras que los lineamientos adoptados por los gobiernos involucrados en la crisis parecen más próximos a decisiones personales de dirigentes políticos con una ideología común (Venezuela, Argentina y Brasil) que a una real factibilidad de los proyectos.

Este contexto político a escala regional explica en parte el inicio de un segundo escenario en el año 2008,también durante el auge de gobiernos progresistas, que lleva a la inserción de la región en el mercado global de la energía con la incorporación de actores extraregionales como abastecedores del recurso gas, en forma de gas natural licuado (GNL), a pesar de la existencia de las mayores reservas de gas en Venezuela y reservas significativas en Perú y Bolivia, dejando de lado no solo la expansión de la integración gasífera regional, sino también aumentando la dependencia y disminuyendo la seguridad energética regional. 
A partir del año 2008 se producen cambios en el transporte pasando del gasoducto a la importación del recurso gas mediante barcos metaneros que transportan gas natural licuado (GNL), se instalan plantas de regasificación (flotantes y terrestres) en distintos países de la región (Brasil, Chile,Argentina) y una planta de licuefacción en Perú con la particularidad de que no exporta a la región. Así, la Región Sudamericana se incorpora como mercado emergente al mercado global del GNL, pero las nuevas instalaciones impactan principalmente, a escala local, en los territorios donde se instalan las plantas (Guerrero,2014). Se observa así cómo los cambios en los procesos de integración energética atraviesan todas las escalas.

En el año 2008 también se descubren hidrocarburos en aguas profundas y ultraprofundas del presal en Brasil, que luego de un desarrollo tecnológico propio comienzan a explotarse. Desde una perspectiva política y económica se fortalece la posición de este país en su disputa regional con Venezuela y consolida su posición como actor global. Brasil pasa de ser un país autosuficiente a ser superavitario en materia energética. A partir del año 2012, se descubren hidrocarburos no convencionales en la formación Vaca Muerta (Argentina). Según los estudios realizados por Advanced Resources International en 2013, la Argentina ocupa el segundo lugar a escala global en shale gas y el cuarto en shale oil.

De cara al futuro, el posible desarrollo de los recursos gasíferos no convencionales de Vaca Muerta en 2019, sacaría a la Argentina de la crisis de abastecimientode gas que inició el conflicto en 2004. Sin embargo, en 2020, la situación provocada por la baja del precio del petróleo sumada a la disminución de la demanda a escala global como consecuencia del COVID-19, genera un panorama muy incierto para lograr atraer inversiones que favorezcan la recuperación de este incipiente desarrollo, más allá del volumen de sus reservas de gas y petróleo. Además la Argentina se encuentra en otra encrucijada: todas las medidas tienden a incrementar la presencia del gas en la matriz energética nacional y no impulsan su diversificación como se propone para alcanzar la Transición energética.

En la actualidad, según un informe de CEPAL de 2019, en América Latina se destaca el uso del gas natural que fue acompañado por una política de penetración del mismo en la matriz energética de los países de la región. En Sudamérica, salvo en el caso de Uruguay y Paraguay, el resto de los países poseen reservas probadas de este recurso que ascienden a 7.528 miles de millones de m3. 
La producción de gas en la región se ubicó en el año 2016 en 178,8 miles de millones de m3 (representando el $5 \%$ de la producción mundial). El cociente entre el volumen de reservas y la producción $(\mathrm{R} / \mathrm{P})$, para el conjunto de países sudamericanos, arroja un valor de 42,1 años (levemente por debajo de la media mundial, del orden de 52,4 años, según informan Di Sbroiavacca et al. (2019).

En el año 2020 este escenario continúa desarrollándose con dinámicas diversas que llevan a la Región Sudamericana a alejarse de la posibilidad de expansión de la integración gasífera a pesar de poseer abundantes reservas. Dicha integración emerge con una dinámica de conflictos que muestran cómo las decisiones políticas y económicas tomadas por los países de la región beneficiaron el consumo del GNL, que favorece la inserción al mercado global de la energía pero debilita la expansión de la integración gasífera regional.

Asimismo, en el período 2004 - 2019 se observa la presencia de un número creciente de actores, regionales y extraregionales, en una trama cada vez más densa que aviva la incertidumbre debido a la convivencia de situaciones - contrapuestas y simultáneas de cooperación y conflicto entre los distintos procesos de integración política, económica y energética. Por lo tanto, a pesar de la existencia de abundantes reservas de gas, la región no se autoabastece y es vulnerable a los vaivenes del precio del petróleo y del gas como bien sustituto.

Además, desde el punto de vista ambiental a pesar que, como algunos sostienen, el gas natural es un combustible puente para la transición energética debido a sus emisiones de carbono más bajas en comparación con otros combustibles fósiles, no es, sin embargo, una solución de carbono cero. Asimismo, también puede variar en función de si se consume localmente a través de un gasoducto o si es convertido a GNL y transportado a largas distancias y luego vuelto a transformar, ya que implica un mayor consumo de energía.

\section{III.2. INTEGRACIÓN ELÉCTRICA}

Los recursos energéticos en general y la energía eléctrica en particular, como sostiene Percebois (2013)

Presentan atributos de carácter estratégico y connotaciones de índole geopolítica, que los diferencian de los demás bienes. Poseen una doble 
dimensión: bien estratégico y servicio público, por ello los Estados o Regiones, buscan controlar o supervisar el acceso, desarrollo y funcionamiento de estos mercados y de la infraestructura de interconexión para el comercio transfronterizo de energía eléctrica (como citado en Levy et al., 2020, p.25).

Para analizar la integración eléctrica en la Región Sudamericana se toman tres informes: a) BID 2019, específico sobre la temática de la Integración eléctrica regional y dos más más generales; b) Organización Latinoamericana de Energía (OLADE), Prospectivas Energéticas 2018 y; c) informe de energía Bp 2019.

Los sistemas eléctricos latinoamericanos se han desarrollado esencialmente durante la segunda mitad del siglo XX con la construcción de plantas de generación que primero abastecían a comunidades más cercanas, mediante sistemas aislados que dependían de la disponibilidad y calidad de su central eléctrica. Luego, resultó evidente la necesidad de integración de los sistemas aislados a nivel nacional mediante la incorporación de redes de Transmisión Eléctrica, que permitían conectar comunidades y generadores distantes, tendiendo a un único Sistema Interconectado Nacional. Posteriormente, surge la integración eléctrica entre países vecinos como posibilidad cuando sus sistemas de transmisión se van acercando a la frontera permitiendo la interconexión cuando se genera energía a menor costo en otro país, o por la falta de capacidad de generación en uno y los excedentes en el otro país (Levy et al., 2020).

El informe del BID 2019 sostiene que América Latina ha hecho avances considerables en materia de integración energética, principalmente eléctrica y que, en general, estas interconexiones responden a esfuerzos binacionales y una minoría ha sido producto del esfuerzo del sector privado. Levy (2020) señala que la interconexión eléctrica regional representa una oportunidad para nuestros países. En la gran mayoría de los casos, estudios previos concluyen que los potenciales beneficios justifican los costos, que las inversiones son rentables y que la integración energética se puede convertir en un elemento dinamizador de la economía cuando estos intercambios responden a la racionalidad económica.

Sin embargo, este avance no ha sido lineal y el aprovechamiento de las ventajas y beneficios de la integración eléctrica es inferior al potencial que ofrece la región. De acuerdo con datos del BID2019 sobre el comercio de electricidad en la región en el 
período 2005 a 2017, se observa que Paraguay, con las centrales binacionales Itaipú y Yaciretá, es el mayor exportador de electricidad en la región alcanzando valores cercanos al $80 \%$ del total. En cuanto a la importación, el $70 \%$ del total de la electricidad la importa Brasil que le compra a Paraguay energía de la central binacional Itaipú. Un caso similar ocurre con la Argentina, quien le compra la porción de la energía que le corresponde a Paraguay de la central binacional Yaciretá y otra parte a la central binacional de Salto Grande, situada entre la Argentina y Uruguay.

En la región ANDINA, Colombia, Ecuador, Perú, Chile y Bolivia han acordado el impulso del Sistema de Interconexión Eléctrico Andino (SINEA). La misma solo cuenta con interconexiones bilaterales entre Colombia-Ecuador y Ecuador-Perú que operan principalmente en casos de emergencia y para intercambio de excedentes con reglas de mercado spot. Colombia, Ecuador, Perú y Chile cuentan con sistemas de transmisión nacionales robustos en $500 \mathrm{KW}$ que podrán ser la columna vertebral que enlazará las nuevas interconexiones binacionales SINEA. Al 2017, la capacidad instalada en los países del SINEA es de 62000 MW con una demanda pico de aproximadamente 34000 MW (Levy et al., 2020:15).

En el CONOSUR (Argentina, Brasil, Paraguay y Uruguay) existen varias interconexiones binacionales: Chile-Argentina (una única interconexión); ArgentinaBrasil (dos interconexiones con sus conversores de frecuencia asociadas); ArgentinaParaguay (dos pequeñas interconexiones) y la central hidroeléctrica binacional Yaciretá de $3200 \mathrm{MW}$, con capacidad de intercambio de $1550 \mathrm{MW}$ desde Paraguay a la Argentina y $750 \mathrm{MW}$ de la Argentina a Paraguay; Brasil - Paraguay (un pequeña interconexión de 50MW) y la central hidroeléctrica binacional Itaipú, con una potencia instalada de $14000 \mathrm{MW}$. En esta región no existe un mercado regional que fomente un mayor intercambio de energía más allá de transacciones binacionales comprometidas por acuerdos previos. Al 2017, la capacidad instalada en estos países alcanza 192000 MW con una demanda pico promedio de 113000 MW (Levy et al., 2020:16).

Estos datos, en relación con el comercio y la interconexión eléctrica, implican un vínculo físico permanente entre los sistemas interconectados internacionales y constituyen un potencial factor de integración entre los países. Sin embargo, existen barreras que lo impiden que pueden surgir de la percepción que tienen los Estados sobre la seguridad de suministro de energía (dimensión geopolítica de la energía: 
soberanía y seguridad energética), la cual aparece como una de las principales barreras a la integración.

La desconfianza y la incertidumbre entre los Estados de la región aparece asociada con amenazas que pueden provenir del país exportador por posible inestabilidad política o por desastres naturales. Ello hace que no se generen vínculos fuertes entre los países y que se vea a esa posible dependencia como una amenaza a su seguridad puesto que ciertas fallas o eventos producidos en un país pueden tener efectos sobre otro. Ejemplo de esta situación es el caso de la Argentina cuando, a pesar de contratos firmados y gasoductos construidos con Chile, ante problemas de abastecimiento prioriza su mercado interno.

Otro factor de preocupación se relaciona con la seguridad jurídica, por ello, las reglas de intercambio deben ser estables a fin de otorgar previsibilidad, pero a la vez, deben tener el margen de flexibilidad necesario para adaptarse a circunstancias cambiantes puesto que los esquemas regionales de integración energética en Latinoamérica son muy diversos, se originan por diferentes razones y presentan elevadas disparidades en sus niveles de avance, como sostiene el informe del BID 2019.

En definitiva, como sostienen Levy et al (2020)

La preocupación sobre la seguridad de suministro se ha convertido en un obstáculo para que los mercados energéticos regionales y las interconexiones binacionales se puedan convertir en fuentes de suministro firme de energía, que permitan aprovechar los beneficios de economías de escala en proyectos de generación más grandes y el acceso a fuentes más económicas de generación (p.27).

Por último, se observa que la integración eléctrica en la Región Sudamericana, en comparación con la integración gasífera, aparece como más estable, con menos conflictos y menor incidencia de los cambios políticos, aunque tampoco se ve una expansión del sistema de interconexión eléctrico ni presencia de acuerdos multilaterales. Como se mencionó, tal vez los gobiernos y la sociedad recuerden su dependencia de la electricidad, evidenciado en la pandemia del COVID-19, y comiencen a revalorizar la importancia de disponer de energía eléctrica en forma confiable y asequible para lo cual es importante contar con infraestructura energética. 
Desde la dimensión ambiental y en el marco de la transición energética, sería deseable que la región avance en un mayor proceso de desfosilización, disminuyendo la participación del gas en la matriz eléctrica e incrementando la participación de las nuevas energías renovables, eólica y solar. De este modo contribuiría también con el cambio climático y el desarrollo sostenible.

\section{INCERTIDUMBRES EN LA TRANSICIÓN ENERGÉTICA SUDAMERICANA}

En este apartado cabe preguntarse si la transición energética sudamericana se encuentra alineada con la que se está produciendo a escala global. En relación con el recurso gas, resulta un componente fundamental de la transición global y de la transición energética dual, como la denomina el informe Bp 2019, por considerarlo el combustible puente hasta tanto se alcance el desarrollo de las energías renovables a mayor escala. En la Figura 2, sobre la base del informe del BID 2018, se puede observar cómo, entre 1980 y la proyección para el año 2030, en la matriz energética regional fue perdiendo peso la participación del petróleo y creció la participación del gas, así como la escasa participación del carbón. A futuro, se espera incrementar la participación de las nuevas energías renovables (eólica y solar) y los biocombustibles.

Figura 2. Matriz de Generación de electricidad por combustible en América Latina,1980-2030, en \%

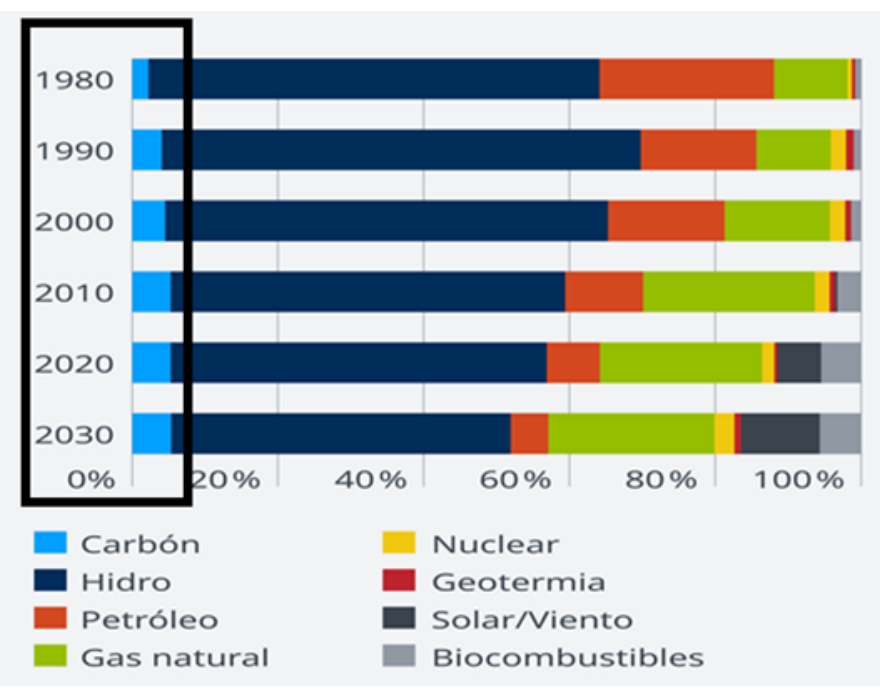

Fuente: Banco Interamericano de Desarrollo, 2018

El Informe presentado por la Organización Latinoamericana de Energía (OLADE), Prospectivas Energéticas 2018, sostiene que, tanto en el año 2016 como en la 
proyección al año 2040, las mayores fuentes de producción de energía eléctrica en la región provienen de la hidroelectricidad y del gas como también se observa en la Figura 2. La energía hidroeléctrica representa el $44 \%$ de la producción de energía eléctrica en el año 2016 y se proyecta que para el año 2040 representará el 37\%. Cabe señalar que la energía hidráulica no se considera dentro de las nuevas energías renovables por considerar que no es sustentable por su impacto en el ambiente, puesto que la construcción de grandes represas implica a veces desplazar a pueblos completos de sus territorios, alterar el curso de los ríos, lo cual produce pérdida en la biodiversidad de la zona y puede generar pérdida de valor del paisaje. Sin embargo, desde el punto de vista de la contaminación del aire no afecta al ambiente.

En relación con otras fuentes de energía renovables, se resalta en particular el uso creciente de la energía eólica, que pasaría del 3\% en el año 2016 al $12 \%$ en el año 2040, mientras que la energía solar alcanzaría a representar solo el 3\% del total (Castillo et al., 2018, 384). En cuanto a la producción de energía eléctrica a partir del gas, representa el $28 \%$ en el año 2016 y se proyecta que se incrementará al 30\% en el año 2040. El siguiente gráfico (Figura 3), permite observar cómo se distribuyen las diversas fuentes de combustibles para la generación de electricidad en las distintas regiones del mundo. Se resaltan en la Región Sudamericana la hidroelectricidad y el gas como los principales componentes, con escasa presencia del carbón.

\section{Figura 3. Generación regional de electricidad por combustible, 2018 en \%}

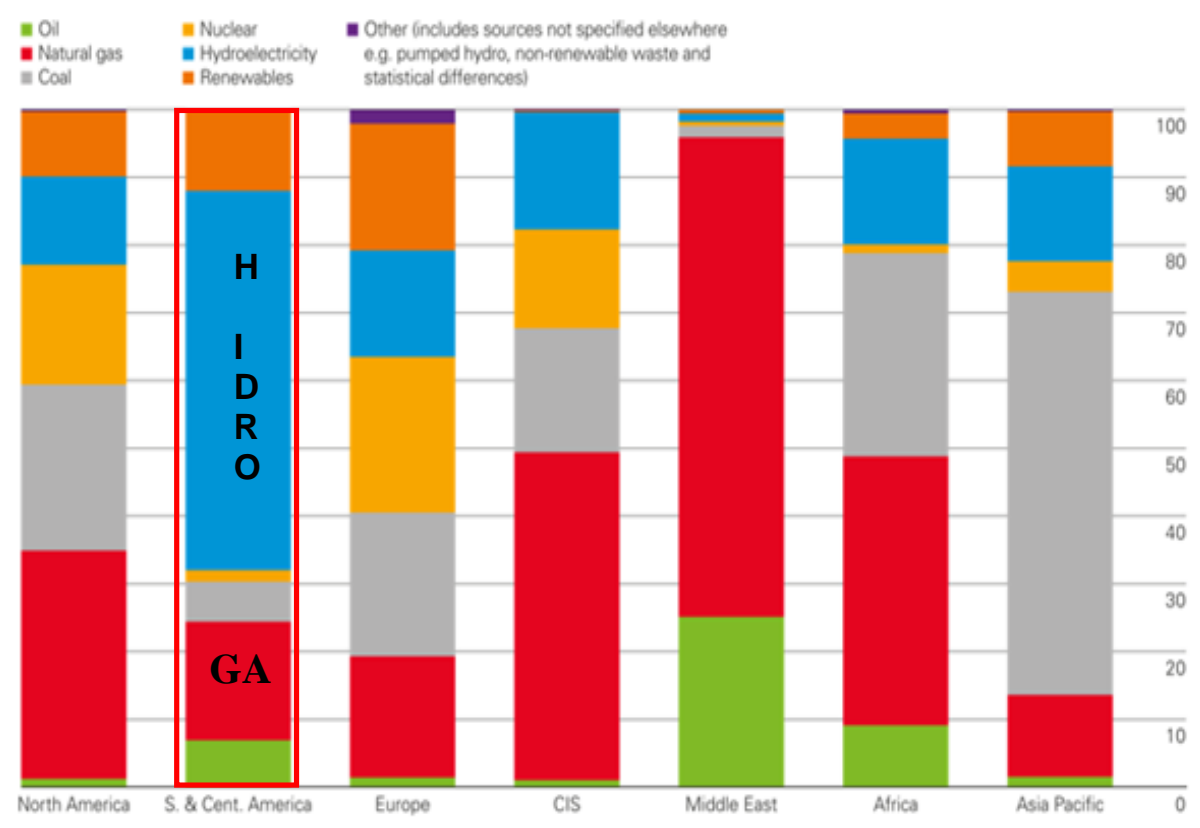

Fuente: Bp Statistical Review of World Energy 2019 
En relación con la transición energética sudamericana, se observa que los cambios en la matriz de generación eléctrica, en cuanto a las fuentes de energía empleadas, van en la misma dirección que a escala global con una transición energética dual. Se destaca, como particularidad regional el peso que tiene la energía hidroeléctrica. A ello se suma el cambio que ya realizó la región desde el uso del petróleo hacia un mayor uso del gas como combustible para la generación de electricidad, y la escasa presencia del carbón en comparación con el resto de las regiones del mundo.

Existe una gran incertidumbre provocada por no saber cuándo se alcanzará, tanto a escala global como regional, una mayor presencia de las nuevas energías renovables para concretar la tercera transición energética. Desde una mirada pragmática, considerando que lo fundamental es disminuir los contaminantes, se puede afirmar que en la Región Sudamericana, desde el punto de vista ambiental, la matriz eléctrica regional es más descarbonizada y menos contaminante que las matrices de energía eléctrica de otras regiones del mundo. Con el tiempo, sería deseable avanzar hacia una matriz desfosilizada de carbono 0 , con mayor presencia de las nuevas energías renovables, como la eólica y solar, a fin de alcanzar una integración energética regional sostenible.

\section{REFLEXIONES FINALES}

El análisis realizado permite observar cómo la integración es un campo de acción multidimensional (político, económico, social, ambiental entre otros) que se apoya en tres pilares fundamentales: la integración económica (comercio), la integración política (relaciones entre los Estados) y la integración física (infraestructura de transporte y energética). El crecimiento económico implica flujos de energía y recursos que constituyen la base material de los procesos de integración. Sin embargo, para pensar no solo en crecimiento sino en desarrollo sostenible, es necesario incorporar la dimensión ambiental y social.

Desde este contexto, el estudio de la integración energética (focalizada en integración gasífera e integración eléctrica) no de forma aislada sino en sus imbricaciones con la integración política/económica regional, en conjunto con la transición energética que incorpora la dimensión ambiental, implica un desafío analítico de mayor alcance y complejidad. En este marco ampliado de la problemática energética regional, el cambio climático continúa siendo el reto global más importante para la sociedad y, 
para actuar sobre él, es necesario considerar escenarios sostenibles, desde la perspectiva ambiental que promueve la transición energética.

En este sentido, la región se encuentra bien posicionada a escala global puesto que, tanto su matriz de generación de energía primaria como su matriz eléctrica se apoya principalmente en la hidroelectricidad y el gas con escasa participación del carbón y, por lo tanto, se encuentra en una transición energética dual como ocurre a escala global; sin embargo, debe avanzar hacia una mayor incorporación de las nuevas energías renovables, eólica y solar.

El incremento de la participación de estas energías en la Región Sudamericana debe considerar que si bien el costo de las fuentes sol, viento y agua es cero, el costo final de la tecnología está ligado a otros factores y variables de tipo técnico, regulatorio, fiscal y de política energética que estimulen inversiones en energías renovables, con tecnología propia, donde los Estados son actores centrales para la toma de decisión.

Como sostienen Paikin et al. (2016), la realidad muestra que en la Región Sudamericana existen limitaciones burocráticas en cada país así como dificultades para entregar soberanía y pensar en objetivos supranacionales como un bien mayor. En este contexto, los continuos vaivenes en los procesos de integración política/económica regional sudamericana en las primeras décadas de este siglo, donde se siguen creando bloques regionales, llevan a plantearse, desde la perspectiva de la integración energética sudamericana, si esta situación de incertidumbre en el rumbo a seguir provoca que se fragmenten en múltiples acuerdos bilaterales y no se generen alianzas estratégicas, profundas y estables (Guerrero, 2018).

El problema que afronta hoy la Región Sudamericana no es la falta de recursos renovables y no renovables, ya que existen en diferentes países de la región, sino las consecuencias de las decisiones políticas de uso de algunos recursos, tal el caso del gas, que llevaron a dinamizar sus mercados regionales pero con una mayor dependencia de actores extraregionales. Además, este mercado se encuentra muy relacionado con el eléctrico, por lo tanto, no solucionar los problemas de la integración gasífera dificulta también avanzar en la eléctrica.

En definitiva, continúa vigente, desde el punto de vista de la integración energética, la pregunta que ya hizo Honty en 2006 cuando cuestionaba si había ¿integración regional o interconexión energética?, es decir, interconexión sin integración. La 
respuesta parece continuar siendo la misma puesto que los procesos de integración no son lineales, presentan avances y retrocesos. En este sentido, más de una década después no se observan avances, y se continúa presenciando una interconexión física -que no se ha expandido- para transportar electricidad y gas natural, más que una integración energética regional que incorpore también la dimensión ambiental.

Desde el punto de vista de la infraestructura física de la integración energética, la Región Sudamericana presenta incertidumbre en cuanto a la posibilidad de ampliar las interconexiones tanto gasíferas como eléctricas y alcanzar acuerdos multilaterales, principalmente por la existencia de conflictos históricos irresueltos, falta de inversiones en infraestructura, desconfianza entre los países de la región para ceder soberanía y disputas por el liderazgo regional, donde la energía no aparece como elemento de integración sino de poder para quien la posee y, por lo tanto, como factor de disputa.

Desde el punto de vista ambiental y del análisis multiescalar se observa cómo la agenda global permea las decisiones regionales para avanzar en relación con el cambio climático, el desarrollo sostenible y la transición energética. Para alcanzar a futuro una integración energética sostenible en la cual confluyan los ODS 7(energía asequible y no contaminante),12 (acción por el clima) y 17 (alianzas para lograr los objetivos), se deberá aumentar el uso de energías renovables en diferentes sectores; serán necesarias inversiones públicas y privadas en energía así como mayores niveles de financiación y políticas que articulen la integración, la facilitación de procesos y el desarrollo tecnológico propio, a fin de generar una agenda común de Transición Energética Regional que lleve a alcanzar un Desarrollo Energético Regional Sostenible.

De este modo, se procura alcanzar no solo una integración energética, sino una transformación del sistema energético en su conjunto a fin de otorgar previsibilidad, pero a la vez, mantener el margen de flexibilidad necesario para adaptarse a un contexto global y regional incierto, dinámico y complejo.

Por último, desde el punto de vista de la transición energética y el cambio en las fuentes a emplear, los Estados son los responsables de impulsar, de manera directa o indirecta, las inversiones en el sector energético, fomentar un mayor diálogo entre los diferentes actores y generar los mecanismos políticos necesarios. La reducción en los costos de producción de las energías renovables y los avances en tecnologías de 
almacenamiento abren nuevas oportunidades para pensar en una transición energética más profunda. Por ello, la velocidad de adaptación al cambio en los Estados y el liderazgo con visión de futuro es crítica para la región, pero se debe recordar que el logro de resultados favorables para alcanzar una integración energética sostenible y una transformación energética profunda depende de las decisiones que tomemos hoy.

\section{BIBLIOGRAFìA}

British Petroleum. (2019). Bp Statistical Review of World Energy 2019 [White paper]. https://www.bp.com/content/dam/bp/businesssites/en/global/corporate/pdfs/energyeconomics/statistical-review/bp-stats-review-2019-full-report.pdf

Carrizo. S. (2019). Argentina en el vaivén de la integración energética sudamericana entre los siglos XX y XXI. En A. Guerrero, M. De Batista y M. Estrada (Coords.), Desarrollo regional en Sudamérica: Investigaciones $y$ aportes multidisciplinarios (pp.173-191). Ediuns. http://repositoriodigital.uns.edu.ar/handle/123456789/4785

Castillo, T. et al. (2018). Panorama energético de América Latina y el Caribe [White paper]. Organización Latinoamericana de Energía. http://biblioteca.olade.org/opactmpl/Documentos/old0416b.pdf

Comisión Económica para América Latina y el Caribe. (2018). Segundo informe anual sobre el progreso y los desafíos regionales de la Agenda 2030 para el Desarrollo Sostenible en América Latina $y$ el Caribe [White paper]. https://www.cepal.org/es/temas/agenda-2030-desarrollo-sostenible/foro-paisesamerica-latina-caribe-desarrollo-sostenible-seguimiento-regional-la-agenda-2030

Di Sbroiavacca, N., Dubrovsky, H., Nadal, G. y Contreras, R. (2019). Rol y perspectivas del gas natural en la transformación energética de América Latina: aportes a la implementación del Observatorio Regional sobre Energías Sostenibles (Documento de Proyectos LC/TS.2019/23). Naciones Unidas CEPAL. https://repositorio.cepal.org/handle/11362/44596

Fernández, V. (2010). Desarrollo regional bajo transformaciones transescalares ¿Por qué y cómo recuperar la escala nacional? En V. Fernández, y C. Brandao (Eds.), Escalas y políticas del desarrollo nacional. Desafíos para América Latina (pp.301341). Miño y Dávila. 
Fouquet, R. y Pearson, P. (2012). Past and prospective energy transitions: insights from history. Energy Policy, 50, 1-7. https://www.sciencedirect.com/journal/energypolicy/vol/50/suppl/C

Guerrero, A. (2014). El gas Natural licuado y su impacto en la circulación de la energía. Análisis Multiescalar. Revista Transporte y Territorio, (11), 5-32. https://www.redalyc.org/pdf/3330/333032406002.pdf

Guerrero A. (2016). La Nueva Geopolítica de la Energía en la Región Sudamericana. Tendencias, actores y conflictos en la industria del gas [Tesis Doctorado, Universidad Nacional del Sur]. Repositorio institucional -Universidad Nacional del Sur. http://repositoriodigital.uns.edu.ar/bitstream/123456789/2944/1/Tesis\%20Doctoral\%20

Guerrero.pdf

Guerrero, A. y Bermann, C. (2019). Visión Geopolítica de los Procesos Espaciales y Dinámicas Territoriales que Reconfiguran la Transición Energética en Sudamérica. En Anais do Seminário Internacional Territórios da energia, mudanças climáticas e sustentabilidade da macrometrópole paulista (p.16). IEE; USP. http://bit.ly/AnaisEnergia

Guerrero, A. (2019). Reconfiguración de la integración en América en entornos geopolíticos dinámicos y complejos. VII Congreso Nacional de Geografía de Universidades Públicas (p.20). Universidad Nacional de La Plata. Facultad de $\begin{array}{lllll}\text { Humanidades } & y & \text { Ciencias la }\end{array}$ http://jornadasgeografia.fahce.un/p.edu.ar/frontpage/actas/ponencias/Guerrero.pdf

Hallack, M. y Yepez, A. (2020, 27 de marzo). Retos del sector de energía con la crisis del Coronavirus. Banco Interamericano de Desarrollo. https://blogs.iadb.org/energia/es/retos-del-sector-de-energia-con-la-crisis-delcoronavirus/

Honty, G. (2006). Energía en Sudamérica una interconexión que no integra. Nueva Sociedad, (204), 119-135. https://nuso.org/articulo/energia-en-sudamerica-unainterconexion-que-no-integra/ 
Howitt, R. (1998). Scale as relation: musical metaphors of geographical scale. Royal Geographical Society, $\quad 30, \quad$ 49-58. ibg.onlinelibrary.wiley.com/loi/14754762/year/1998

Iniciativa para la Integración de la Infraestructura Regional Sudamericana. (2020). Consejo Sudamericano de Infraestructura y Planeaminto. http://www.iirsa.org/Page/PageDetail?id=80\&menultemld=81

Global Commission on the Geopolitics of Energy Transformation. (2019). Global Commission describes geopolitical power dynamics created by renewable [Press release]. International Renewable Energy Agency. https://www.irena.org/newsroom/pressreleases/2019/Jan/Global-CommissionDescribes-New-Geopolitical-Power-Dynamics-Created-by-Renewable-Energy

Levy, A., Tejeda, J., y Di Chiara, L. (2020). Integración eléctrica regional: oportunidades y retos que enfrentan los países de América Latina y el Caribe (Monografía del BID, 742). Banco Interamericano de Desarrollo. http://dx.doi.org/10.18235/0002129

Organización de las Naciones Unidas. (2015). Agenda Para el Desarrollo Sostenible 2030. https://www.un.org/sustainabledevelopment/es/development-agenda/

Paikin, D., Perrotta, D., y Porcelli, E. (2016). Pensamiento latinoamericano para la integración. Crítica y Emancipación, 49-80. http://biblioteca.clacso.edu.ar/clacso/se/20171019112058/CyE N15.pdf

Recalde, M. Y. , y Guzowsky, C. (2016). Política energética y desarrollo socioeconómico: Una aplicación al caso argentino. En C. Guzowsky, (Comp.), Políticas de promoción de las energías renovables. Experiencias en América del Sur (pp.15-56). Ediuns.

Sanahuaja, J. A., y Comini, N. (2018). Las nuevas derechas latinoamericanas frente a una globalización en crisis. Nueva Sociedad, (275), 32-46.

https://nuso.org/articulo/las-nuevas-derechas-latinoamericanas-frente-globalizacion-encrisis/ 
Sanahuja, J.A. (2019). La crisis de la integración y el regionalismo en América Latina: giro liberal-conservador y contestación normativa. En M. Mesa, (Coord), Ascenso del nacionalismo y el autoritarismo en el sistema internacional Anuario CEIPAZ 20182019 (pp. 107-126). CIPAZ; Fundación Cultura de Paz. https://ceipaz.org/wpcontent/uploads/2020/04/ANUARIO-Completo-2018-2019.pdf

Tokatlian J. (2019, febrero). América Latina camina hacia la debilidad y la desintegración. Nueva Sociedad, Opinión. https://nuso.org/articulo/america-latinacamina-hacia-la-debilidad-y-la- desintegracion/

GuerRero, ANA Lía del Valle: Docente Investigadora Departamento de Geografía y Turismo, Universidad Nacional del Sur (UNS). Doctora en Geografía (UNS). Magister en Políticas y Estrategias (UNS). Licenciada y Profesora en Geografía (Universidad de Buenos Aires-UBA). Docente de Posgrado en la Maestría en Políticas y Estrategias (UNS) y en la Maestría en Desarrollo y Gestión Territorial (UNS). Directora del proyecto "Geopolítica y Territorio. Procesos territoriales emergentes en la articulación local - global en América Latina".E-mail: aguerrero@uns.edu.ar 